\title{
EVOLUTIONARY CHANGES IN THE ECLIPSING BINARY $\beta$ LYRAE
}

\author{
P. HARMANEC \\ Astronomical Institute, Acad. of Sciences of the Czech Republic, 25165 Ondrrjov, \\ Bohemia, Czech Republic
}

An attempt at the first direct test of the theory of large-scale mass transfer between the components of a binary system is discussed. It is argued that $\beta$ Lyr is the only binary in the sky for which such a test is conceivable. 